\title{
Investigation on Application of Comparative Teaching Method in the Course of Engineering Graphics
}

\author{
Yanjiao $\mathrm{Li}$ * \\ Xi'an Research Instu of Hi-Tech \\ Hongqing Town, Xi'an, Shanxi, 710025, P. R. China \\ lyjxjtu@126.com \\ Zhiqing Guo \\ Xi'an Research Instu of Hi-Tech \\ Hongqing Town, Xi'an, Shanxi, 710025, P. R. China \\ hahahguo239@163.com
}

\author{
Qiujuan Lv \\ Xi'an Research Instu of Hi-Tech \\ Hongqing Town, Xi’an, Shanxi, 710025, P. R. China \\ qiujuanlv@163.com \\ Chen Cheng \\ Xi'an Research Instu of Hi-Tech \\ Hongqing Town, Xi'an, Shanxi, 710025, P. R. China \\ cche943@sina.com
}

\begin{abstract}
Engineering graphics is a compulsory basic course for engineering students in military academies. The characteristics of the course are abstract and complex, requiring students to have strong spatial thinking ability and image thinking ability. However, because students are in contact with the course for the first time, their thinking ability is still in the course of training. It is difficult to understand some difficult problems and can't grasp the key problem, which leads to difficulties in drawing pictures. In order to solve the difficult problems and improve the students' learning efficiency, in view of some key and difficult problems in the course, this paper analyzes the possible problems that may arise in the course of students' learning, and expounds how to use comparative teaching method to solve these problems and the good results obtained by using this method. This paper uses specific cases to illus trate the exploration and application of comparative teaching method in the course of engineering graphics, which is intuitive and specific, and can provide reference for the teaching design of engine ering graphics and other courses.
\end{abstract} method

Keywords - engineering graphics; comparative teaching

\section{INTRODUCTION}

The course of engineering graphics is a required basic course for the mechanical and related engineering major. It mainly trains the students' spatial thinking ability, image thinking ability and engineering quality. Because the course mainly studies how to express the three-dimensional form on the two-dimensional drawing, it needs to carry out the thinking transformation of the two dimensional figure and the threedimensional form. Therefore, for many students, the course is abstract and difficult to understand. In the process of learning and completing the homework, students often find it difficult to quickly imagine the three-dimensional structure of the twodimensional graphics, especially some difficult structures. In order to reduce the difficulty of students' learning and help students to understand the course content quickly and accurately, teachers should take various effective teaching methods, resolve difficulties, reduce the difficulty of learning and improve the students' learning efficiency.

The comparative method of teaching is an method which means that two or more than two cognitive objects are placed under certain conditions in the teaching activities and compared with the same standard, so as to determine the similarities and differences of the attributes of the object, the size of the position, the size of the function, the quality of the performance, the difficulty of the problem or the truth and error of the recognition, in order to achieve identification, understand and grasp the purpose of knowing objects. The comparative teaching method has the characteristics of the universality of comparative objects, the penetration of comparative thinking, the differentiation of comparative functions and the contrast of comparison methods. The content of comparative teaching method is very rich. The principle that the comparative teaching method follows is to make the knowledge clear and easy to grasp easily and easily. Therefore, the comparative teaching method is not only one of the most commonly used teaching methods, but also one of the most widely used methods of teaching[1-5].

The course of engineering graphics is an abstract and difficult course. Many concepts and structures are difficult to understand or even confuse in the course, which causes the students to make mistakes repeatedly in the course of learning. In the course of teaching, the author fined that the comparative teaching method has a good effect in solving some confusing problems in this course. Based on years of teaching experience, this paper discusses the application of comparative teaching in engineering graphics[6].

\section{The APPLICATION of COMPARATIVE TEACHING}

Method in Line And Surface Projection Teaching

The projection of the straight line and the projection of the plane are the important basic knowledge of the course of engineering graphics, which seems to be simple, but the proficiency in their mastery is directly related to the speed of 
the following drawing. Because there are many similar places between the lines of various positions and between the planes of various positions, if the students fail to distinguish their characteristics, they are often confused when they are applied, leading to the imaginary errors of the projection or the structure of the body. In order to allow students to quickly and accurately grasp the projection law of the straight line and plane, the students were guided to compare with the projection of the lines and planes in various positions, and the key features of the projection law are analyzed by the comparison of the same and different types of projection. On the base of the analysis of the law, a series of contrast exercises were carried out to consolidate and deepen the contents in the minds of students.

For example, the projection law of the vertical plane of the projection plane and the parallel plane of the projection surface can be summed up as "one line two frame line tilt" and "two lines and one frame line parallel". At the same time, the projection law will be used to explain the meaning clearly, helping the students to grasp the projection characteristics of the two special position plane. Many years of teaching practice show that the application of comparative teaching method enables most students to grasp this part firmly and apply them skillfully in the study of follow-up content, which lays a good foundation for the teaching of intersections and composite solids.

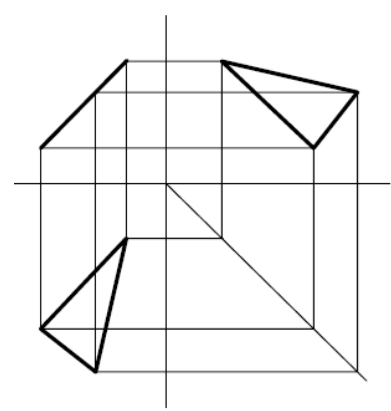

(a)V-perpendicular plane

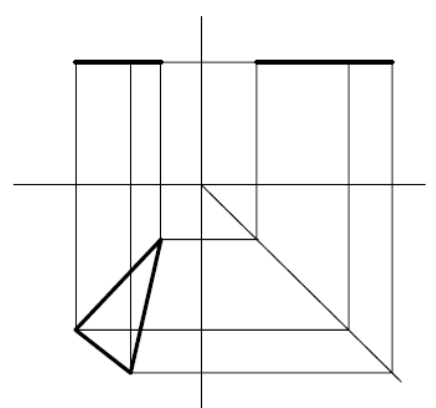

(b)horizontal plane
Fig. 1. Contrast examples of plane projection

\section{The Application of Comparative Teaching}

Method in the Teaching of CylindRICAL Cutting

Cylindrical cutting is a key and difficult problem in the teaching of engineering graphics. When students learn this part, the general drawing method can be mastered quickly, but it is especially easy to make mistakes on some details. As in the structure shown in Figure 2, there are two questions for the students to find out in the left view. First, does the profile of the cylinder exist and exist in what range? Second, is the side projection perpendicular to the axis of the cylinder a dotted line or a solid line? What is the scope of the virtual reality line? If these problems can't be thoroughly clarified, the trainees are prone to make mistakes in solving such problems

In order to clarify this kind of problem and let students have a clear understanding of the problem, comparison examples shown in Figure 2 were set, and then combine the virtual model, make the structure cut range and projection characteristics clear, let the students understand the essence of the problem and thoroughly understand the problems. The setting of this comparison example can help students imagine, and can highlight the difference of the problem, thus reduce the difficulty of the study, increase the students' interest in learning, and achieve a good teaching effect.
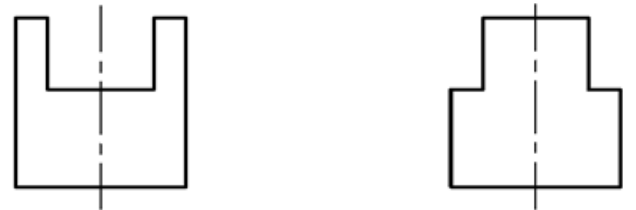

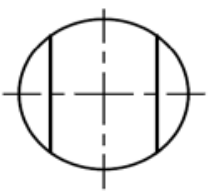

(a)

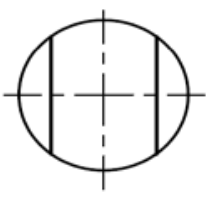

(b)
Fig. 2. Comparison examples of cylindrical cutting

\section{The Application of Comparative Teaching} METHOD IN CYLINDRICAL ORTHOGONAL INTERSECTION LINE TEACHING

Cylindrical orthogonal intersecting line is a difficult problem in the teaching of engineering graphics. Most of the students often find it difficult to imagine their spatial shape and the trend of projection when they learn this part, so they always feel helpless when they are drawing. The key to such problems lies in the fact that students do not fully understand the formation process of intersecting lines.

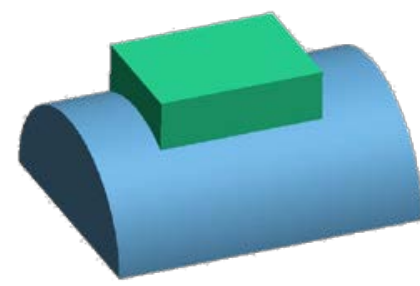

(a) Intersecting model of plane stereoscopic and revolving body

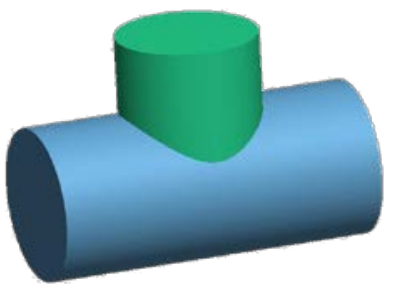

(b) Intersecting model of two revolving bodies

Fig. 3. Inters ecting line contrast diagram

\section{The Application of Comparative Teaching METHOD IN THE TEACHING OF RIB STRUCtURE}

Ribbed slab is a common structure on machine parts. It often appears in the course of engineering graphics. In the course of teaching, the author found that the students' understanding of the intersection of the rib structure and the matrix is not accurate, especially when the rib structure appears 
on both sides of the cylinder or hollow cylinder, because the thickness of the ribs is usually thinner, the outer surface of the rib plate and the cylinder or hollow cylinder structure is smaller, and its shape and position are not obvious. These obscure structural features will lead to errors in their understanding and result in incorrect drawing.
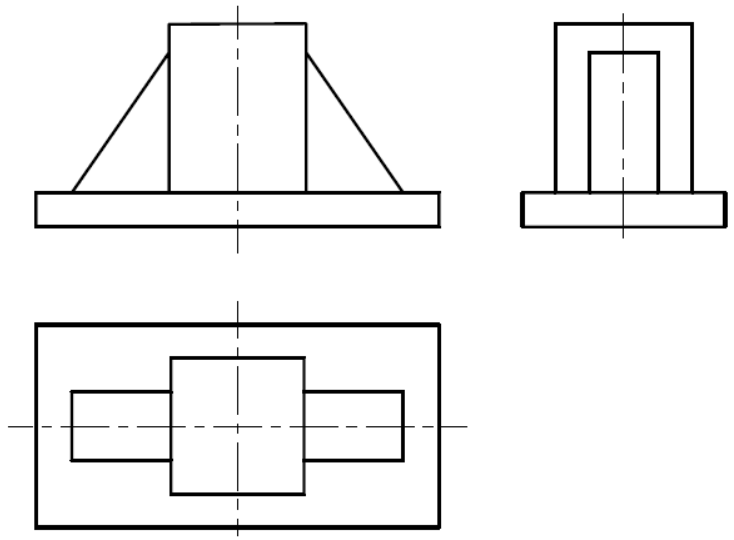

(a) Intersecting model between the ribs and the plane
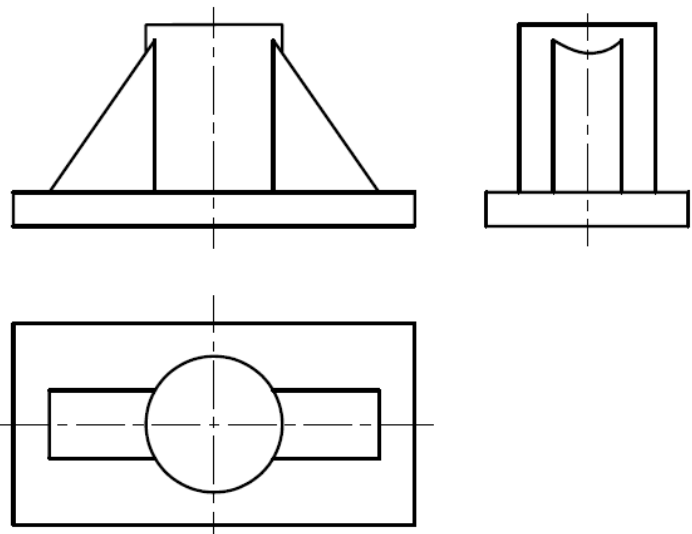

(b) Intersecting model between the ribs and the column

Fig. 4. Contrast diagram of rib structure

In order to guide the students to understand the structure accurately, the intersecting model between the ribs and the plane and the intersecting model between the ribs and the column were established by the three-dimensional modeling software such as SolidWorks, as shown in Fig.4(a), (b). Then the models were rotated and magnified and the students were guided to observe and compare them from different angles, so that the students can recognize the similarities and differences of the two structures and correctly understand their shape and position characteristics. The correctly understand of this structure will lay a solid foundation for rapidly drawing and reading such structures.

\section{Vi. The Application of Comparative Teaching} Method in the Teaching of Cross SeCtion Map

Cross-section is one of the important expression methods of machine parts. In learning and applying this expression method, students are easily confused with sectional views and do not know how to draw and apply the cross-section correctly. In order to solve this problem, a comparative teaching method is adopted to compare the concept, representation of cross-section and sectional view to guide students to recognize the similarities and differences and the application scope of the two expression method. As shown in Figure 5, through the contrast of the picture, the students are given a cross section map that only draws the shape of the section, and the sectional view is to draw all the structures behind the section. By emphasizing "only drawing" and "drawing all the structures" to deepen the students' understanding of the similarities and differences of the two expression method, and thus understand the difference of the concept and apply it in the drawing process.

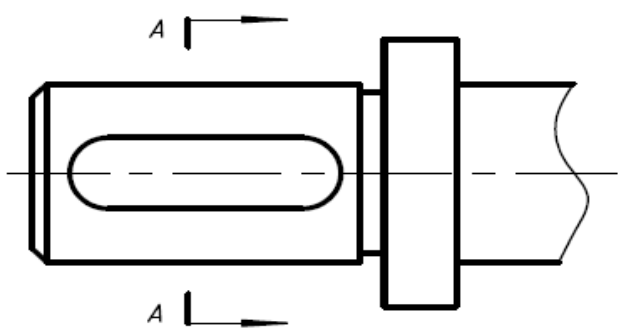

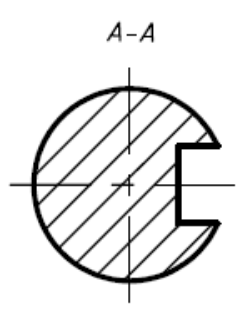

(a)Cross-section

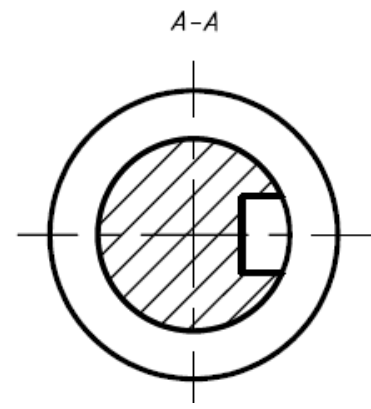

(b) Sectional view
Fig. 5. The difference between Cross-section and sectional views

VII. The Application of Comparative Teaching Method IN THE TEACHING OF AXONOMETRIC DRAWING

Axonometric drawing is a relatively simple and attractive content in graphics course. Most students like to learn this part, and can better grasp its drawing method. In spite of this, some students are prone to make some mistakes due to the obfuscation of the axes when they measuring the length of the side along the axis, which leads to the poor expression of some cutting structures after the completion of the axonometric drawing.

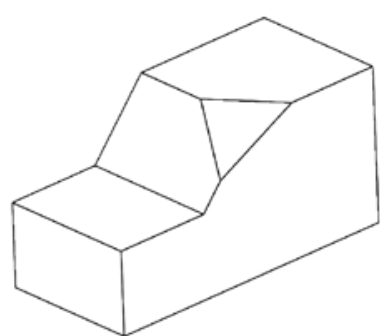

(a)

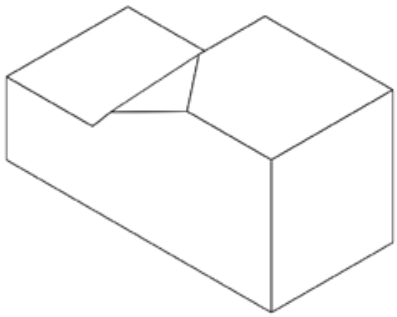

(b)
Fig. 6. Axonometric drawing with different angles of cutting type combination 
In order to explain this situation to the students, through the comparative teaching method, the results of the drawing of the two coordinate axes are displayed to show the results of the students to compare their effect, so as to understand the importance of the coordinate axis and to take the correct value of the coordinate values.

In order to explain this situation to the students, a comparative teaching method can be used to show the drawing results adobe from the selection of two different coordinate axes, so that the students can compare their effects by themselves and understand the importance of the correct selection of coordinate axis and to take the correct value of the coordinate values. Figure 6 is an axonometric drawing of a cut combination of different angles. It can be seen that figure (a) is a complete representation of the cut structure, while figure (b) is not clearly expressed in the cut structure. Part of the structure can't be completely presented because of the display angle problem, and it is obvious and easy to see that figure (a) is better than figure (b) in expressing the stereoscopic structure.

How can we avoid the occurrence of figure (b) ? We can guide students to compare and analyze the amount of coordinate axes, and find that the problem is confused with $\mathrm{X}$ and $\mathrm{Y}$ axis. This contrastive analysis can show the problem directly, analyze the root of the problem, and make the students find the problem and solve the problem in the easy analysis and contrast process. Many years of teaching practice shows that such comparative analysis can achieve good results and the quality of the students' work and the rate of examination are obviously improved.

\section{SUMMARY}

Years of teaching practice has proved that the comparative teaching method is an effective teaching method, which is very applicable to many problems in the course of graphics. Through comparison, some unintelligible and unimaginable problems can be solved into simple and easy to understand problems, so as to clear the students' learning disabilities, stimulate the interest of the students and improve the quality and effect of the classroom teaching. The rational use of comparative teaching method in the teaching process of engineering graphics courses allows students to explore in comparison, to find laws in comparison and to draw conclusions. This process can cultivate not only students' ability to observe and analyze problems, to find problems and to solve problems, but also the ability to think independently and sum up. Teachers should rationally design the teaching process according to the different characteristics of the course content, and adopt this method at the appropriate time to improve the classroom teaching effect.

\section{ACKNOWLEDGMENT}

Thanks to the fund support of the foundation of Xi'an Hi Tech Research Institute.

\section{REFERENCE}

[1] Y. Li, "Comparative teaching method", Journal of South Central Institute of Nationalities (Hu man ities), vol. 3, pp. 124-126, July 2000.(In Chinese)

[2] X.T. Leng, M.H. Cai, W.Y. Pang. The practical exploration of the three elements of comparative teaching method in higher education, higher education forum. vol. 7 (2), pp. 70-73, March 2014, (In Chinese)

[3] M.R. Guo, A.H. Hao, D.H. Xia, "The application of comparative teaching method in experimental teaching of engineering fluid mechanics". Chinese education outside school, vol.7 (2), pp. 71,116, July 2014.(In Chinese)

[4] Z.H. Liu, "The application of comparative teaching method in the construction and maintenance of engineering machinery chassis", Journal of Liaoning Institute of Science and Technology, vol. 2 (2), pp. 49-50, June 2010, (In Chinese)

[5] K.M. Yang, X.L. Yang, “Application of comparative teaching method in the teaching of water supply engineering”. Higher education research, vol. 27 (2), pp. 71-73, June 2010.(In Chinese)

[6] Y.L. Cao, Y.Q. Wang, B.L. Ren. "Comparative law applied research in mechanical drawing teaching”. University Education, pp. 161-162, May 2016.(In Chinese) 